\title{
Inter-Communicative Decentralized Multi-Scale Control (ICD-MSC) Scheme: A New Approach to Overcome MIMO Process Interactions
}

\begin{abstract}
Decentralized PID control has been extensively used in process industry due to its functional simplicity. But designing an effective decentralized PID control system is very challenging because of process interactions and dead times, which often impose limitations on control performance. In practice, to alleviate the detrimental effect of process interactions on control performance, decoupling controllers are often incorporated into a decentralized control scheme. In many cases, these conventional decoupling controllers are not physically realizable or too complex for practical implementation. In this paper, we propose an alternative scheme to overcome the performance limitation imposed by process interactions. This new control scheme is extended from the SISO multi-scale control scheme previously developed for nonminimum-phase processes. The salient feature of the new control scheme lies in its communicative structure enabling collaborative communication among all the sub-controllers in the system. This communicative structure serves the purpose of reducing the detrimental effect of process interactions leading to improved control performance and performance robustness. Extensive numerical study shows that the new control scheme is able to outperform some existing decentralized control schemes augmented with traditional decoupling controllers.
\end{abstract}

Keywords: multi-loop PID control, decentralized control, multivariable process, multi-scale control, decoupling control

*Corresponding author: Jobrun Nandong, Department of Chemical and Petroleum Engineering, Curtin University Sarawak, Miri, Sarawak 98009, Malaysia, E-mail: jobrun.n@curtin.edu.my

Zhuquan Zang, Department of Chemical and Petroleum Engineering, Curtin University Sarawak, Miri, Sarawak 98009, Malaysia, E-mail: zqzang@curtin.edu.my

\section{Introduction}

Most industrial processes are multi-input and multi-output (MIMO) systems, where the applications of multi-loop
PID controllers (decentralized control schemes) are very common due to their simplicity in implementation [1]. Nevertheless, the performance of decentralized PID controllers in MIMO processes is often limited by the presence of process interactions. For this reason, several decoupling techniques have been proposed to overcome the detrimental effect of process interactions on control performance and performance robustness. Among the decoupling techniques, the conventional decoupling types are the most commonly used in process industry.

The methods for designing multi-loop PID controllers can be broadly categorized into five major groups [2]: (1) detuning, (2) sequential loop closing, (3) iterative or trial-and-error, (4) simultaneous equation solving, and (5) independent methods. The detuning approach is commonly used due to its simplicity. One of the well-known detuning methods is based on the biggest log-modulus [3]. The idea of BLT detuning method is to first design an individual PI/ PID controller for each loop using some established SISO PID tuning procedures, e.g. Ziegler-Nichols procedure [4]. Then, upon closing all of the control-loops, the PI/PID controllers are detuned via adjusting a common parameter until the specified BLT value is attained. Although the BLT method is quite easy to use, the resulting closed-loop responses are often too sluggish or oscillatory; but the BLT tuning results can be further improved via a dominant pole placement method of Lee and Edgar [5].

The sequential design method is based on the idea, which is to design an individual PID controller one at a time, followed by closing the loop involved. Subsequently, another PID controller is designed with the first PID controller already in active mode. This procedure of designing a PID controller and closing the loop sequentially is repeated until all loops are closed. The sequential design approach was introduced in 1970s [6] and has been quite extensively studied, e.g. Shiu and Hwang [7], Hovd and Skogestad [8], and Loh et al. [9]. One of the common disadvantages of the sequential loop closing is that the final control system performance might not be satisfactory 
because the order of the loop closing often affects the control performance overall. In other words, one has to find the right sequence of loop closing in order to obtain good overall control performance. Unfortunately, for many real multivariable processes determining the right order of loop closing is often not obvious.

The independent approach might be able to resolve the poor control performance resulting from the sequential design. The advantage of an independent design method is that it can guarantee the failure tolerance by satisfying the inequality constraints on the process interactions [10]. Several design methods have emerged based on the independent design approach, e.g. independent method based on effective open-loop transfer functions [11], one-parameter method [12], and method based on Nyquist stability analysis [13].

It should be noted that many of the design methods for the multi-loop PI/PID controllers are based on process models, i.e. the methods are analytic in nature. Here, the internal model control (IMC) approach is often used to systematically perform the PID controller design, e.g. the method of $\mathrm{Vu}$ and Lee [11] and Lee et al. [14]. In cases where process models are unavailable, one may revert to using the relay autotuning approach; see e.g. Astrom and Hagglund [15] and $\mathrm{Yu}$ [16]. The relay feedback method is quite simple to use for obtaining the frequency information for PI controller design.

Meanwhile, there are three main categories of the conventional decoupling types known as: (1) ideal decoupling, (2) simplified decoupling, and (3) inverted decoupling [17]. These different decoupling types have been shown to possess similar robust stability provided that they are designed with identical nominal performance [18]. Among the three types of conventional decoupling, the simplified and ideal decoupling techniques are the most widely used in industry [19]. For the simplified decoupling applied to a $n \times n$ MIMO system, $n$ elements (usually the diagonal elements) of the decoupler matrix $\mathbf{D} \in R^{n \times n}$ are often set to unity.

It is interesting to note that, the simplified decoupling has often been used to develop some of the advanced multivariable control strategies. For example, Garrido et al. [20] proposed a centralized control design, which was based on the simplified decoupling technique. Here, it was shown that the decoupler elements that are set to unity might not be necessarily the diagonal elements. This decoupling method allows for different configurations of decouplers, depending on which elements are set to unity. Rajapandiyan and Chidambaram [21] proposed another simplified decoupling technique that was designed based on the equivalent transfer function (ETF) models of a given MIMO system. The decoupled ETF models were used to design PI/PID controllers via an independent design approach. Based on some numerical examples, this method was demonstrated to be able to provide better performance than the ideal, inverted, and normalized decoupling methods.

Unfortunately, one of the main disadvantages of the ideal and simplified decoupling approaches is that the resulting decoupler elements can be very complex especially for large systems. Consequently, this often leads to physically unrealizable decoupler elements [22]. Motivated by this disadvantage some researchers have proposed an improved alternative known as the inverted decoupling. The inverted decoupling technique was shown to possess some practical advantages [17]: (1) ease of initialization that allows for bumpless transfer between manual and automatic modes and (2) incorporation of input saturation handling into the inverted decoupling structure. However, the inverted decoupling technique cannot be applied to MIMO processes which possess right half plane (RHP) zeros. In view of this gap, another improved inverted decoupling technique was proposed by Chen and Zhang [23]. This modified inverted decoupling technique is able to address the multiple time-delays and RHP zeros. Moreover, Garrido et al. [22] presented an extended inverted decoupling method that is applicable to a general $n \times n$ MIMO processes.

In the present paper, we propose an alternative decentralized control scheme capable of overcoming the performance limitation imposed by the presence of process interactions. This new scheme is called the ICD-MSC, and it is based on the MSC scheme recently proposed by Nandong and Zang [24, 25] for SISO nonminimum-phase processes. The design principle of the MSC scheme is to first decompose a given plant into a sum of basic modes, each having distinct speed of responses. Then, an individual (P/PI) sub-controller is designed to control each of these plant modes. Finally, an overall multi-scale (MS) controller is constructed by combining all of the P/PI subcontrollers in a manner that enhances the cooperation among the different plant modes - this feature is vital to improving control performance and performance robustness. In an ideal case, the MS controller can possess several inner feedback loops (each feedback loop is intended to control one specific inner mode) and with one outermost feedback loop, which controls the slowest (outermost) mode. Note that the inner feedback loop is faster than the outer feedback loop. Hence, the scheme is called the MSC because of the combination of several sub-controllers with different speeds [24, 25].

In the currently proposed ICD-MSC scheme, the fast inner-layer feedback loop (sub-controller) of one MS controller (known as the receiving MS controller) is used to 
make an early compensation for the disturbance (due to process interaction) coming from another MS controller (called the transmitting MS controller). To achieve this goal, the receiving MS controller has to have an access of information on the action made by the transmitting MS controller or vice versa. The control action by the transmitting MS controller is then treated as an input disturbance by the fast inner-layer sub-controller of the receiving control-loop. In other words, the receiving MS controller is allowed to communicate with another transmitting controller, where this communication can take place either in a 1-way or in a 2-way, i.e. either partial or complete inter-communication, respectively. It is interesting to note that, the disturbance due to the action made by the transmitting controller is compensated for by the fast inner-loop of the receiving controller via the inner-layer feedback compensation. In contrast, for the conventional decoupling techniques, the disturbance (i.e. loop interaction) due to one controller is compensated for by a decoupler acting in a manner of feedforward control action; the decoupler is often considered as a special case of feedforward control.

The rest of the paper is structured as follows. Section 2 provides a brief summary of the MSC scheme for a SISO process. In Section 3, the idea of how to extend this basic MSC scheme to the proposed ICD-MSC scheme for a case of $2 \times 2$ MIMO processes is presented. Furthermore, two structures of the ICD-MSC scheme are described, and a number of methods for obtaining the required inter-communicative controller are proposed. Section 4 provides a general design procedure for the ICD-MSC scheme. Then in Section 5, the applicability of the ICD-MSC scheme is demonstrated using some practical examples (including one example based on a nonlinear multivariable fermentation system). Finally, conclusions and some future studies are highlighted in Section 6.

\section{Summary of the multi-scale control scheme}

The basic idea of the MSC scheme proposed by Nandong and Zang $[24,25]$ is to first decompose a given plant $P$ into a sum of basic modes, each having distinct speeds of responses - MS dynamics. For a plant which can be decomposed into a sum of $n+1$ basic modes, i.e.

$$
P(s)=M_{0}(s)+M_{1}(s)+M_{2}(s)+\cdots+M_{n}(s)
$$

where $M_{i}, \forall i=\{0,1,2, \ldots, n\}$ is a basic mode which can be either a first- or a second-order transfer function with real coefficients. Note that $M_{i}$ is assumed to be slower than $M_{i+1}$ for $i=0,1, \ldots n-1$. Here, $M_{0}$ is called the outermost mode while $M_{i}$ the inner-layer mode for $i=1, \ldots, n$.

As an illustration, let us consider a plant that is decomposable into a sum of two basic modes only $\left(P=M_{0}+M_{1}\right)$. Hence, two separate sub-controllers $\left(K_{0}\right.$ and $K_{1}$ ) are needed where each one is specifically designed to control each of the plant modes, i.e. $K_{1}$ is used to control the inner-layer mode $M_{1}$ and $K_{0}$ to control $M_{0}$. To achieve good overall control performance, it is vital that the two sub-controllers be assembled in a way that they function cooperatively. This can be done by a cascaded combination where the fast mode is used as a slave to the slower mode as shown in Figure 1.

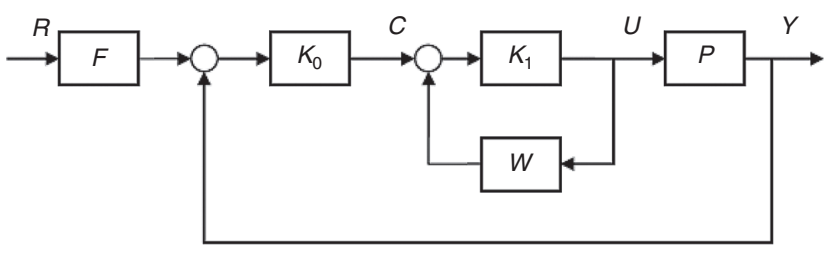

(a)

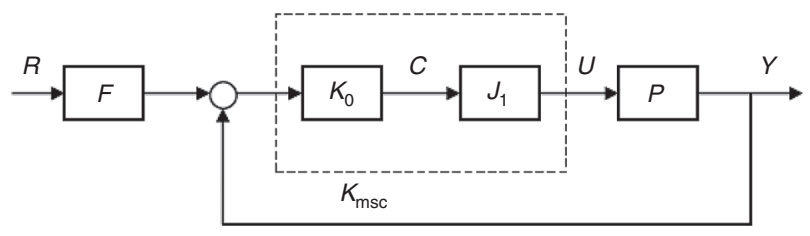

(b)

Figure 1 The MSC scheme: (a) two-layer MSC and (b) equivalent single-loop feedback control

With respect to Figure 1, $P$ denotes the plant to be controlled; $F$ the setpoint pre-filter; $W$ the MS predictor; $K_{j}, j=1,2$ the sub-controller; $R$ the setpoint; $U$ the manipulated input; $D$ the input disturbance; $Y$ the controlled output. Note that, the two-layer MSC scheme (Figure 1(a)) can be reduced to an equivalent singleloop feedback control as shown in Figure 1(b).

Based on Figure 1(b), the closed-loop inner-layer transfer function is given by

$$
J_{1}(s)=\frac{U(s)}{C(s)}=\frac{K_{1}(s)}{1+K_{1}(s) W(s)}
$$

where the MS predictor is often chosen as the fast inner mode

$$
W(s)=M_{1}(s)
$$

The overall MS controller could be expressed as follows:

$$
K_{\mathrm{msc}}(s)=K_{0}(s) J_{1}(s)
$$


Meanwhile, the augmented overall plant transfer function is given by

$$
P_{c}(s)=J_{1}(s) P(s)
$$

where the setpoint closed-loop transfer function is

$$
H_{r y}(s)=\frac{Y(s)}{R(s)}=\frac{F(s) K_{\mathrm{msc}}(s) P(s)}{1+K_{\mathrm{msc}}(s) P(s)}
$$

and the input disturbance closed-loop transfer function

$$
H_{d y}=\frac{Y(s)}{D(s)}=\frac{P(s)}{1+K_{\mathrm{msc}}(s) P(s)}
$$

\section{The proposed ICD-MSC scheme}

\subsection{Preliminary}

Consider a $2 \times 2$ system with a transfer function matrix as given by

$$
\mathbf{G}(\mathbf{s})=\left[\begin{array}{ll}
g_{11}(s) & g_{12}(s) \\
g_{21}(s) & g_{22}(s)
\end{array}\right]
$$

Assuming that the direct pairing (i.e. diagonal transfer functions are used to design the controllers) is adopted and the diagonal transfer functions can be decomposed into a sum of two basic modes, i.e.

$$
g_{i i}(s)=m_{i, 0}(s)+m_{i, 1}(s) \quad i=1,2
$$

So, the MS predictors are chosen as

$$
\mathbf{W}(\mathbf{s})=\left[\begin{array}{l}
w_{1,1}(s) \\
w_{2,1}(s)
\end{array}\right]=\left[\begin{array}{l}
m_{1,1}(s) \\
m_{2,1}(s)
\end{array}\right]
$$

The sub-controller matrix is written as follows:

$$
\mathbf{K}(\mathbf{s})=\left[\begin{array}{ll}
K_{1,0}(s) & K_{1,1}(s) \\
K_{2,0}(s) & K_{2,1}(s)
\end{array}\right]
$$

where $K_{i, j}$ indicates the sub-controller corresponding to the plant mode $m_{i, j}$, i.e. $i$ th control-loop and $j$ th mode of transfer function $g_{i i}$.

\subsection{The input-input (I-I) structure of ICD-MSC scheme}

Figure 2 shows the block diagram of a $2 \times 2$ MIMO system which employs an inter-communicative structure, i.e. ICD-MSC scheme. It is interesting to point out that a distinguishing feature of the scheme from the conventional decentralized control scheme (augmented with a conventional decoupling structure) lies in the inter-communicative controller introduced from the control-loop 2 to control-loop 1, i.e. $I_{12}$. The inter-communicative controller shown in Figure 2 enables the MS controller $K_{\mathrm{msc}}$ (receiving controller) to access the control information (input movement) generated by the MS controller $K_{\mathrm{msc} 2}$ (transmitting controller). The transmitted information from $K_{\mathrm{msc} 2}$ is used by the receiving controller $K_{\mathrm{msc1}}$ to make a pre-adjustment to compensate for the detrimental effect of control action made by $K_{\mathrm{msc} 2}$ on the controlled variable $Y_{1}$. Note that, the main idea underlying this proposed scheme is to treat the control movement made by $K_{\mathrm{msc} 2}$ as an input disturbance on $Y_{1}$, where this disturbance is to be removed via the fast feedback loop of $K_{\mathrm{msc} 1}$ before it can significantly affect $Y_{1}$. In this case, this pre-compensation action is performed by the inner-layer sub-controller $K_{1,1}$ (the fast feedback loop of $K_{\mathrm{msc}}$ ) in a manner of feedback control. Hence, for this strategy to be

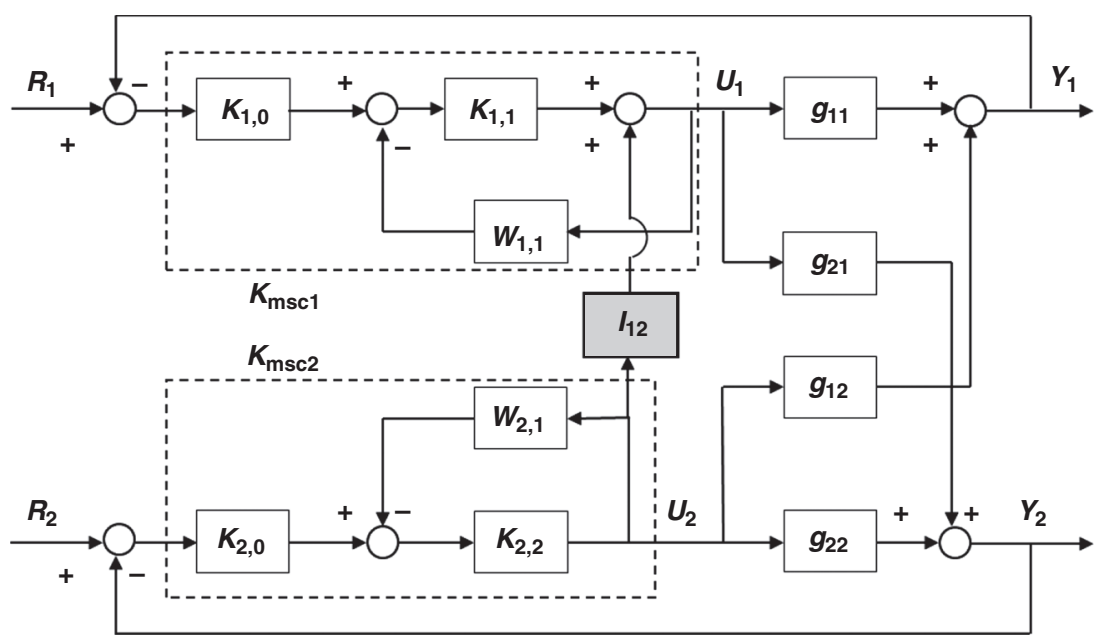

Figure 2 The 1-way I-I structure of ICD-MSC scheme 
effective, the inner sub-controller of $K_{\mathrm{msc} 1}$ must be responsive enough to the effect of the disturbance generated by $K_{\mathrm{msc} 2}$ on $Y_{1}$.

The scheme shown in Figure 2 is referred to as the I-I structure of ICD-MSC scheme. Here, the information from the transmitting controller $K_{\mathrm{msc} 2}$ is extracted at the input point w.r.t. the MS predictor block $w_{2,1}$; the information is sent to the input point w.r.t. the MS predictor block $w_{1,1}$.

Note that, the compensation of the "disturbance" due to $K_{\mathrm{msc} 2}$ on $Y_{1}$ by $K_{\mathrm{msc} 1}$ is in fact achieved by means of a feedback control action of the fast sub-controller $K_{1,1}$ (within the control-loop 1). Bear in mind that the proposed scheme does not attempt to remove the effect of loop interactions in the same sense as the conventional decoupling controller does, which basically follows the principle of feedforward control. Since the proposed inter-communicative scheme uses feedback control principle to mitigate the detrimental effect of loop interactions, the proposed scheme is likely to be more robust against plant modeling errors than the traditional decoupling control scheme, where the effect of interactions among the loops are reduced in a manner of feedforward control.

Referring to Figure 2, for the inner-layer control-loop 1 , the transfer function from $U_{2}$ to $U_{1}$ is given by

$$
g_{12}^{i}=\frac{U_{1}}{U_{2}}=\frac{I_{12}}{1+K_{1} W_{1}}
$$

Here, the superscript " $i$ " is used to indicate the I-I structure of ICD-MSC scheme.

The transfer function that combines the overall effect of $U_{2}$ on $Y_{1}$ via $g_{12}$ and $g_{12}^{i}$ is given as follows:

$$
Q_{12}=\frac{Y_{1}}{U_{2}}=g_{12}^{i} g_{11}+g_{12}
$$

which can be further written in the form of

$$
Q_{12}=\frac{I_{12} g_{11}+\left(1+K_{1,1} w_{1,1}\right) g_{12}}{1+K_{1,1} w_{1,1}}
$$

In general, the inter-communicative controller $\left(I_{12}\right)$ can be obtained by solving the optimization problem given as follows:

$$
\mathcal{P}_{\mathbf{1}}: Q_{12}^{*}=\min _{I_{12}}\left\|\frac{I_{12} g_{11}+\left(1+K_{1} W_{1}\right) g_{12}}{1+K_{1} W_{1}}\right\|_{2}
$$

where the solution is given by $I_{12}=I_{12}^{*}$.

An alternative problem can be formulated which is based on the minimization of integral absolute error (IAE) value of $Y_{1}$ in response to a step change in $U_{2}$. This problem can be stated as follows:

$$
\mathcal{P}_{\mathbf{2}}: Y_{1}^{*}=\min _{I_{12}} \int_{0}^{t_{\mathrm{s}}}\left|\mathcal{L}^{-1}\left(Q_{12} U_{2}\right)\right| d t
$$

where $\mathcal{L}^{-1}$ denotes the inverse of Laplace transform and $t_{s}$ represents the longest settling time of the control-loops in the system.

For simplicity, we may assume a "perfect compensation" at a steady-state, i.e. $Q_{12}=0$, which reduces the problem of solving $\mathcal{P}_{1}$ above to

$$
\frac{I_{12}^{s S} k_{p, 11}+\left(1+K_{1,1} k_{1,1}\right) k_{p, 12}}{1+K_{1,1} k_{1,1}}=0
$$

where $k_{1,1}$ denotes the gain for $w_{1,1}, k_{p, 11}$ the process gain for $g_{11}$, and $k_{p, 12}$ the process gain for $g_{12}$. Solving the above equation leads to a static inter-communicative controller

$$
I_{12}^{S S}=-\frac{K_{p, 12}\left(1+K_{1,1} k_{1,1}\right)}{K_{p, 11}}
$$

For practical implementation, we can set the inter-communicative controller within the range

$$
I_{12}=\alpha_{12} I_{12}^{s s} \quad \alpha_{12} \in\left[\begin{array}{ll}
0.1 & 5
\end{array}\right]
$$

The reason for this suggested range of tuning value is to take into account the dynamics difference between the diagonal and off-diagonal transfer functions.

Note that the ICD-MSC scheme shown in Figure 2 can be readily extended to a 2-way communication structure, i.e. complete inter-communication between the two MS controllers involved.

\subsection{The output-input (0-I) structure of ICD-MSC scheme}

Figure 3 shows a 2-way ICD-MSC scheme for a $2 \times 2$ MIMO system. Here, the two inter-communicative controllers are added, so that both MS controllers involved have a simultaneous access to information on the control movements generated by both of them. It is important to point out that the main difference between this structure and that of the previous I-I structure only lies in the location from which the information on the other controller is extracted. For this scheme, the information is extracted from the output point after the MS predictor block $\left(w_{1,1}\right.$ or $\left.w_{2,1}\right)$ as shown in Figure 3. The extracted information from one control-loop is sent to the input point w.r.t. the MS predictor in another (receiving) MS controller. Hence, this structure is termed as the O-I structure of ICD-MSC scheme.

Interestingly, both of the proposed schemes of ICD-MSC are governed by the same principle, i.e. the information on the action of one (transmitting) MS controller is treated as an input disturbance by the other receiving MS controller. This 


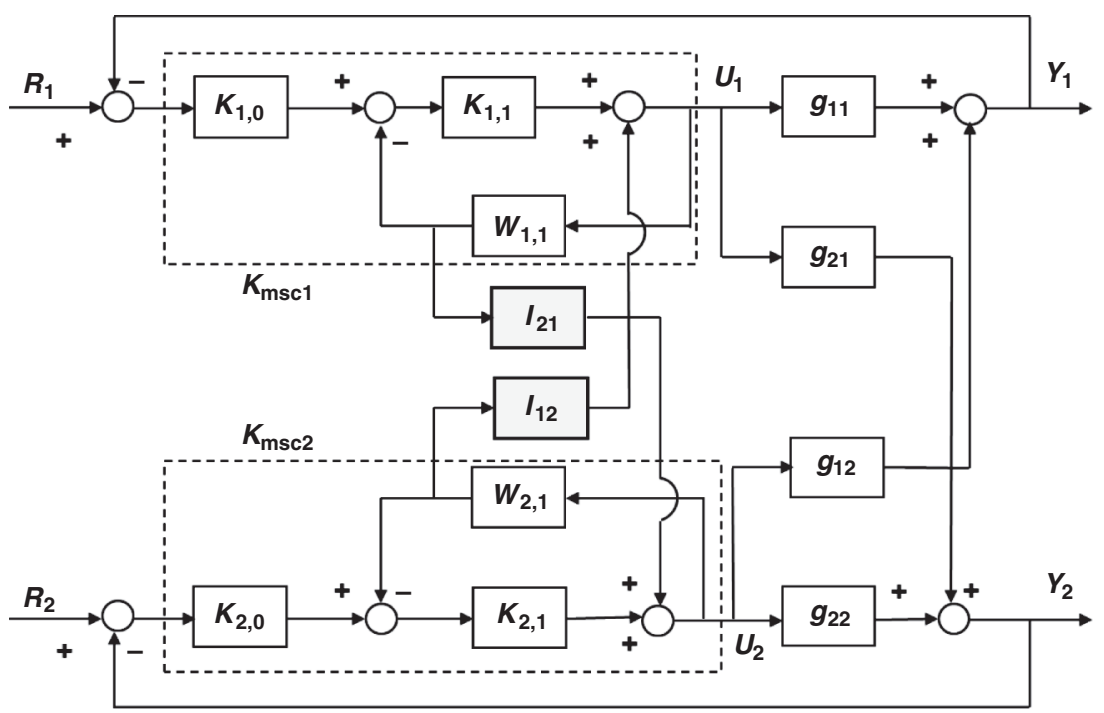

Figure 3 The 2-way 0-I structure of ICD-MSC scheme

disturbance triggers the inner-layer sub-controller of the receiving MS controller to perform an early pre-compensating action to remove or mitigate the effect of the control action generated by the transmitting MS controller.

For a given control-loop shown in Figure 3, the transfer function from $U_{2}$ to $U_{1}$ is given by

$$
g_{12}^{o}=\frac{U_{1}}{U_{2}}=\frac{w_{2,1} I_{12}}{1+K_{1,1} w_{1,1}}
$$

where the superscript " $O$ " indicates the $\mathrm{O}-\mathrm{I}$ structure.

Likewise, we can obtain the transfer function from $U_{1}$ to $U_{2}$ as

$$
g_{21}^{o}=\frac{U_{2}}{U_{1}}=\frac{w_{1,1} I_{21}}{1+K_{2,1} w_{2,1}}
$$

The combined effect of $U_{2}$ on $Y_{1}$ via $g_{12}^{o}$ and $g_{12}$ is expressed as

$$
Q_{12}=\frac{\left(1+K_{1,1} W_{1}\right) g_{12}+I_{12} w_{2,1} g_{11}}{1+K_{1,1} w_{1,1}}
$$

and $U_{1}$ on $Y_{2}$ via $g_{21}^{o}$ and $g_{21}$ as

$$
Q_{21}=\frac{\left(1+K_{2,1} w_{2,1}\right) g_{21}+I_{21} w_{1,1} g_{22}}{1+K_{2,1} W_{2,1}}
$$

In general, the inter-communicative controller $I_{i j}$ for a given control-loop can be obtained by solving the following optimization problem:

$$
\mathcal{P}_{3}: Q_{i j}^{*}=\min _{I_{i j}}\left\|\frac{\left(1+K_{i, 1} w_{i, 1}\right) g_{i j}+I_{i j} w_{j, 1} g_{i i}}{1+K_{i, 1} w_{i, 1}}\right\|_{2}
$$

Also, the inter-communicative controller $I_{i j}$ can be obtained by minimizing the IAE value of the controlled variable $Y_{i}$ in response to a step change in the input $U_{j}$. This problem can be stated as follows:

$$
\mathcal{P}_{4}: Y_{i j}^{*}=\min _{I_{i j}} \int_{0}^{t_{s}}\left|\mathcal{L}^{-1}\left(Q_{i j} U_{j}\right)\right| d t
$$

Similar to the previous I-I structure, upon assuming perfect compensation, i.e. $Q_{i j}=0$, the static inter-communicative controller can be derived as follows:

$$
I_{i j}^{S S}=-\frac{\left(1+K_{i} k_{i, 1}\right) k_{p, i j}}{k_{j, 1} k_{p, i i}}
$$

For a simple implementation, the inter-communicative controller can be set according to the suggested range mentioned in the previous I-I structure.

\section{Design procedure}

We assume that (1) the transfer function matrix is arranged in such a way that the diagonal elements are used to design the MS controllers - direct pairing based on the relative gain array (RGA) analysis, (2) each diagonal transfer function is decomposable into two basic modes, and (3) independent controller design is assumed for each control-loop and where retuning might be necessary upon closing all the loops.

Under the above-mentioned assumptions, the following general procedure can be used to design the subcontrollers for the ICD-MSC scheme.

Step 1: Decompose the diagonal transfer functions $g_{i i}$ into a sum of basic factors or modes as given by eq. (9). 
Note that, the time-delay components can be first approximated by rational transfer functions using the well-known Padé formula.

Step 2: Select the MS predictors as in eq. (10). Use the MS predictor $m_{i, 1}$ to design the sub-controller $K_{i, 1}$.

Step 3: Construct the augmented overall transfer function $P_{c, i}$ for each loop $i=1,2, \ldots, n$ as in eq. (5). Then, use the augmented overall transfer function $P_{c, i}$ to synthesize the outermost sub-controller $K_{i, 0}$.

Step 4: Select a pair of control-loops which are to be linked via the communication structure. Assuming perfect compensation, the inter-communicative controller can be designed based on eq. (18) or (26); a refined tuning value can be chosen within the range given in eq. (19).

Step 5: Close all the control-loops, including the communicative channels. The controllers can be further retuned to give improved performance in terms of IAE values.

\section{Remarks:}

i. The inner sub-controller is chosen as a P-only controller for simple tuning. The tuning can be done using the IMC tuning available in the Matlab SISO Design Tool/Matlab Control System Tuning. For an initial tuning, the dominant closed-loop time constant is chosen to be between $1 / 4$ and $1 / 8$ of the open-loop settling time of a given mode $m_{i, 1}$.

ii. The outermost sub-controller is chosen as a PI/PID controller. The controller tuning can be performed using the IMC tuning, where the closed-loop time constant is set between $1 / 4$ and $1 / 12$ of the openloop settling time of a given mode $m_{i, 0}$.

\section{Illustrative examples}

In this section, we shall demonstrate the effectiveness of the proposed ICD-MSC scheme via some practical examples reported in the open literature.

Example 1 - Industrial-scale polymerization (ISP) reactor. The nominal model of the ISP reactor [26] is given by

$$
\mathbf{G}=\left[\begin{array}{cc}
\frac{22.89 e^{-0.2 s}}{4.572 s+1} & \frac{-11.64 e^{-0.4 s}}{1.807 s+1} \\
\frac{4.689 e^{-0.2 s}}{2.174 s+1} & \frac{5.8 e^{-0.4 s}}{1.801 s+1}
\end{array}\right]
$$

The steady-state RGA for eq. (27) is given as:

$$
\mathbf{R G A}=\left[\begin{array}{ll}
0.71 & 0.29 \\
0.29 & 0.71
\end{array}\right]
$$

Based on the RGA obtained from eq. (27), we can draw a conclusion that the ISP system has significant process interactions. Thus, the application of decentralized control strategy would not be able to give satisfactory performance.

For this example, the performance of ICD-MSC scheme is compared with that of the decentralized control augmented with a simplified decoupling scheme of Rajapandiyan and Chidambaram [21]. They have shown that their scheme can outperform even the centralized control system as well as the ideal, inverted, and normalized decoupling control methods.

\subsection{ICD-MSC scheme}

$$
\mathbf{W}=\left[\begin{array}{c}
\frac{-1.024}{0.1 s+1} \\
\frac{-1.451}{0.2 s+1}
\end{array}\right]
$$

Here, we demonstrate the applicability of both I-I and OI structures of ICD-MSC scheme to the ISP process (27). We choose a 1-way communication of I-I structure. The controller design procedure presented in Section 4 is adopted. The final tunings for the sub-controllers involved are given as follows:

$$
\mathbf{K}=\left[\begin{array}{cc}
\frac{-0.190\left(0.1 s^{2}+5 s+3.5\right)}{s} & -1.1 \\
\frac{-0.456\left(0.05 s^{2}+2.56 s+1.8\right)}{s} & -0.979
\end{array}\right]
$$

where the steady-state inter-communicative controller is $I_{12}^{S S}=1.1$; the implement value is chosen as $I_{12}=2.8$. Note that, the communication channel is from loop 2 (transmitting loop) to loop 1 (receiving loop), i.e. to reduce the disturbance made by the controller 2 on control-loop 1 .

Meanwhile, for the $\mathrm{O}-\mathrm{I}$ structure, we choose a 2-way inter-communication where the final sub-controller tunings are given by

$$
\mathbf{K}=\left[\begin{array}{cc}
\frac{-0.2133\left(0.25 s^{2}+5 s+6\right)}{s} & -1.815 \\
\frac{-0.684\left(0.1 s^{2}+1.8 s+1\right)}{s} & -0.979
\end{array}\right]
$$

Here, the steady-state inter-communicative controllers are $I_{12}^{s s}=-1.5$ and $I_{21}^{s s}=1.1$. The implemented inter-communicative controllers are $I_{12}=-2.5$ and $I_{21}=0.5$.

Additionally, the following setpoint pre-filters are used for both I-I and O-I structures of ICD-MSC scheme.

$$
\left[\begin{array}{l}
F_{1} \\
F_{2}
\end{array}\right]=\left[\begin{array}{c}
\frac{1}{0.1 s+1} \\
\frac{1}{0.4 s+1}
\end{array}\right]
$$

To evaluate the performance robustness of the different control schemes, we consider a perturbed condition for 
the model (27), which is based on 50\% modeling errors in gains of the diagonal elements; $-50 \%$ modeling errors in gains of the off-diagonal elements; and 50\% modeling errors in time-delays of diagonal and off-diagonal elements. The performance of the ICD-MSC scheme is compared with that of the decentralized PID control scheme of Rajapandiyan and Chidambaram [21] with complete (2way) decoupling. Note that, in the Rajapandiyan and Chidambaram decentralized scheme, two decouplers are used to augment two multi-loop PI controllers.

Figure 4 shows the closed-loop responses of the three control schemes at the nominal condition. The 2-way ICDMSC scheme (with O-I structure) shows markedly better performance than the 1-way ICD-MSC scheme (with I-I structure). The IAE values for the ICD-MSC (2-way), ICDMSC (1-way), and Rajapandiyan and Chidambaram schemes for the first controlled variable $Y_{1}$ are 0.78 , 1.27, and 0.81, respectively; for $Y_{2}$ are $0.93,0.97$, and 0.98 , respectively. Hence, based on the IAE values it can be concluded that all schemes show almost similar performance for the second controlled variable. For the first controlled variable, the 2-way ICD-MSC shows similar performance as the complete decoupling scheme.

Figure 5 demonstrates the closed-loop responses in the presence of 50\% modeling errors. It is interesting to highlight that the 1-way and 2-way ICD-MSC schemes show very little performance degradation as compared to the Rajapandiyan and Chidambaram decentralized PI controllers with complete decoupling scheme. This demonstrates that the proposed ICD-MSC scheme is more robust against modeling errors or plant uncertainties than the conventional decentralized controllers with complete decoupling design. Thus, it can be concluded that the proposed ICD-MSC scheme has better performance robustness than the decentralized PID controllers augmented with complete decoupling scheme.

Example 2 - Tyreus distillation column. The nominal model for the Tyreus distillation column is given in Shiu and Hwang [8]:

$$
\mathbf{G}=\left[\begin{array}{ccc}
\frac{1.986 e^{-0.71 s}}{66.7 s+1} & \frac{-5.24 e^{-60 s}}{400 s+1} & \frac{-5.984 e^{-2.24 s}}{14.29 s+1} \\
\frac{-0.0204 e^{-0.59 s}}{(7.14 s+1)^{2}} & \frac{0.33 e^{-0.68 s}}{(2.38 s+1)^{2}} & \frac{-2.38 e^{-0.42 s}}{(1.43 s+1)^{2}} \\
\frac{-0.374 e^{-7.75 s}}{22.22 s+1} & \frac{11.3 e^{-3.79 s}}{(21.74 s+1)} & \frac{9.811 e^{-1.59 s}}{11.36 s+1}
\end{array}\right]
$$

For this example, we apply the 1-way ICD-MSC with I-I structure. The MS predictors are given as

$$
\mathbf{W}=\left[\begin{array}{c}
\frac{-0.0212}{0.355 s+1} \\
\frac{0.018}{0.34 s+1} \\
\frac{-1.476}{0.795 s+1}
\end{array}\right]
$$

The finalized controller tunings are given by
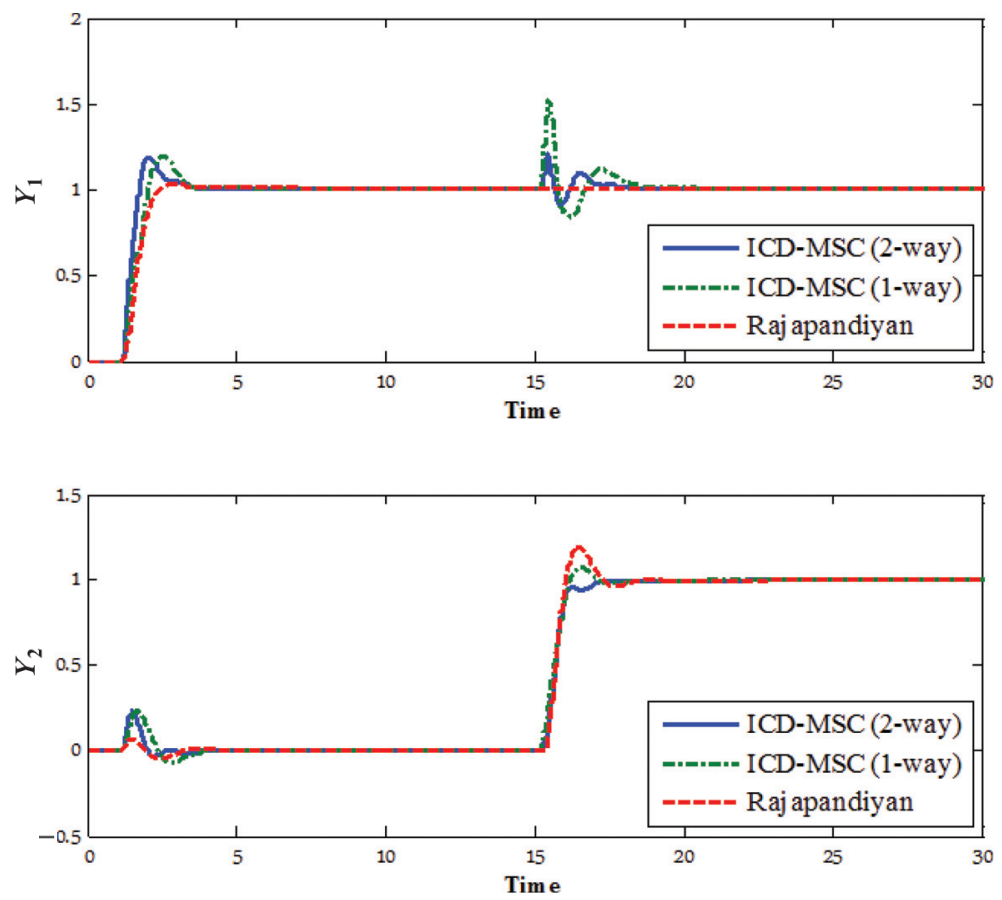

Figure 4 Closed-loop responses at nominal condition for Example 1 

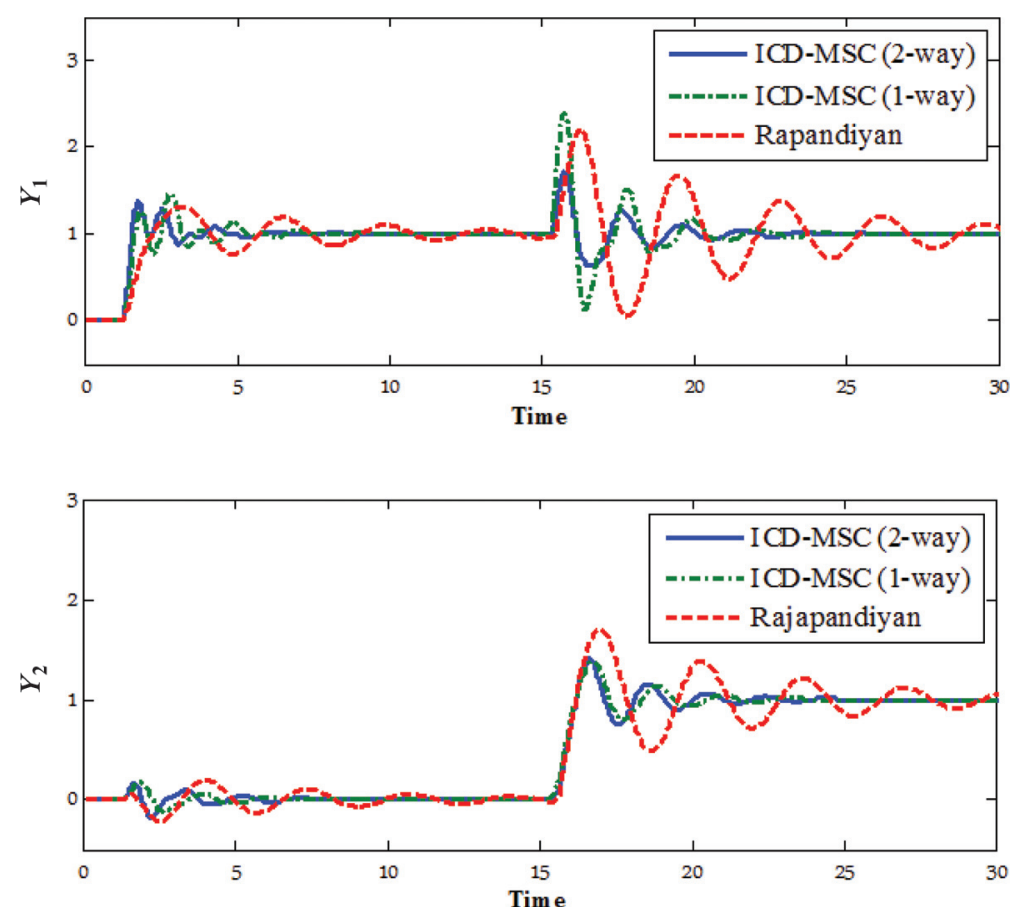

Figure 5 Closed-loop responses at perturbed condition ( $\pm 50 \%$ gain modeling errors $/+50 \%$ time-delay errors) for Example 1

$$
\mathbf{K}=\left[\begin{array}{cc}
\frac{-0.0351\left(0.5 s^{2}+67 s+1.5\right)}{s} & -29.5 \\
\frac{0.0314\left(0.4 s^{2}+4.4 s+0.33\right)}{s} & 50.8 \\
\frac{-0.55\left(s^{2}+5.5 s+1\right)}{s} & -0.29
\end{array}\right]
$$

where setpoint pre-filters are

$$
\left[\begin{array}{l}
F_{1} \\
F_{2} \\
F_{3}
\end{array}\right]=\left[\begin{array}{c}
\frac{1}{2 s+1} \\
\frac{1}{7 s+1} \\
\frac{1}{9 s+1}
\end{array}\right]
$$

Here, we choose a 1-way inter-communication from controlloop 3 to the control-loop 2. The steady-state inter-communicative controller is $I_{23}^{S S}=0.83$. For implementation, we set the inter-communicative controller to be $I_{23}=2.4$. Notice that, only one inter-communicative controller is added to the decentralized MS control scheme.

The performance/robustness of the ICD-MSC scheme is compared with the decentralized control scheme of Shiu and Hwang [8] with partial decoupling structures. In the Shiu and Hwang partial decoupling scheme, three decouplers are used. Thus, in this case the proposed ICDMSC scheme is much simpler than the Shiu and Hwang [8] decoupling multi-loop control scheme.

To evaluate the performance robustness, we consider a perturbed condition in model (32) based on $20 \%$ modeling errors in gains and time-delays for the diagonal elements and $-20 \%$ modeling errors in gains and timedelays for the off-diagonal elements.

Figure 6 illustrates the performance comparisons between the proposed 1-way ICD-MSC scheme with that of the conventional decentralized design of Shiu and Hwang with partial decoupling at the nominal condition. Except for the first controlled variable $Y_{1}$, the proposed ICD-MSC scheme clearly outperforms the Shiu and Hwang decentralized control scheme.

As shown in Figure 7, the proposed ICD-MSC again exhibits better robust performance in the presence of modeling errors than the decentralized control with partial decoupling design. Interestingly, in the presence of $\pm 20 \%$ modeling errors in gains and time-delays, the proposed scheme shows very little closed-loop performance degradation as compared to the decentralized control with partial decoupling. On the other hand, the decentralized control with decoupling design is unstable in the presence of these $\pm 20 \%$ errors in gains and time-delays. This example further highlights that the proposed scheme based on the feedback control action to mitigate the loop disturbance is indeed more robust against plant uncertainties than the conventional decoupling control scheme, which uses feedforward control action to mitigate the loop disturbance.

Example 3-Two-stage extractive continuous fermentation (TSCEF) process. 

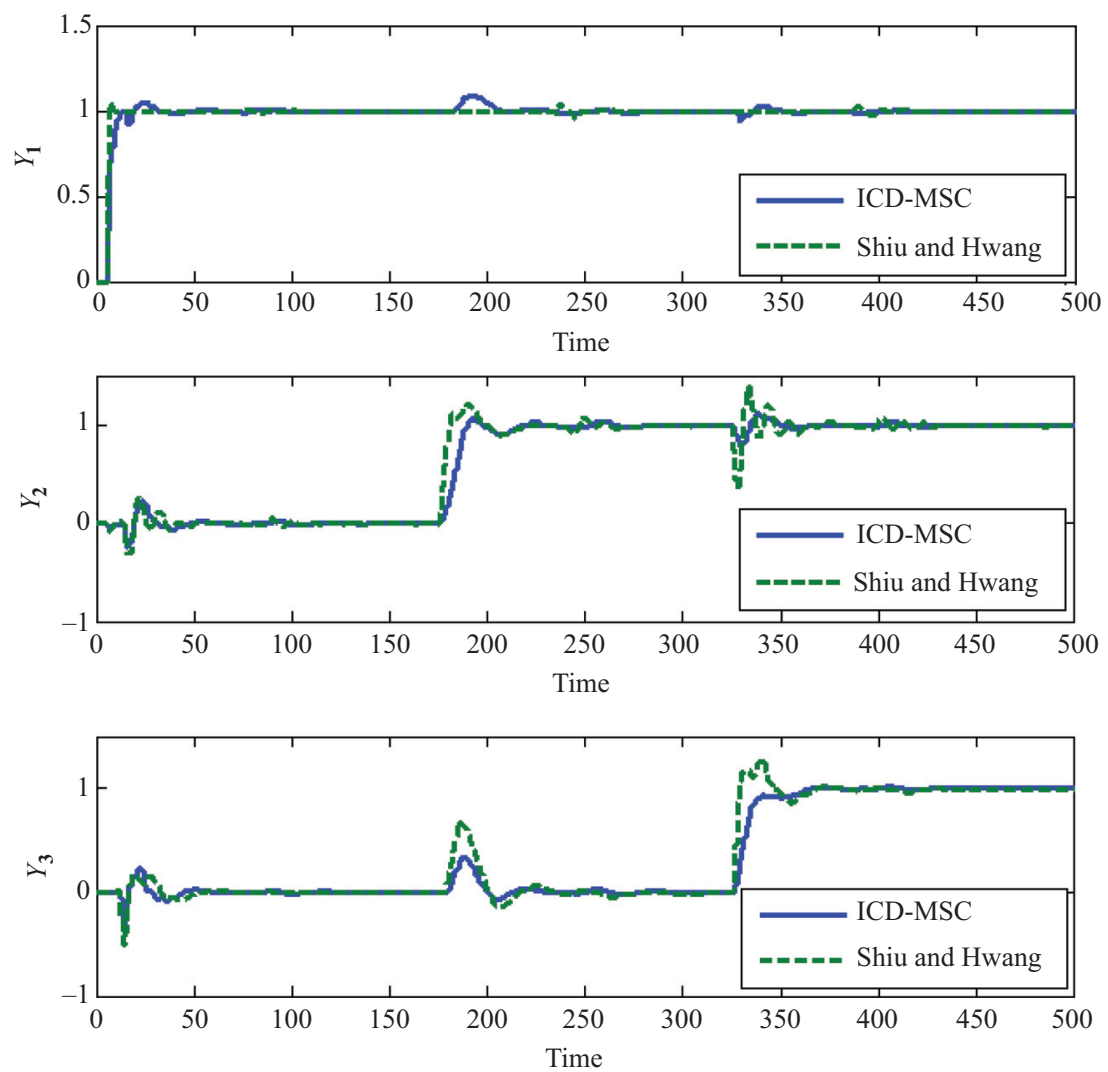

Figure 6 Closed-loop responses at nominal condition for Example 2
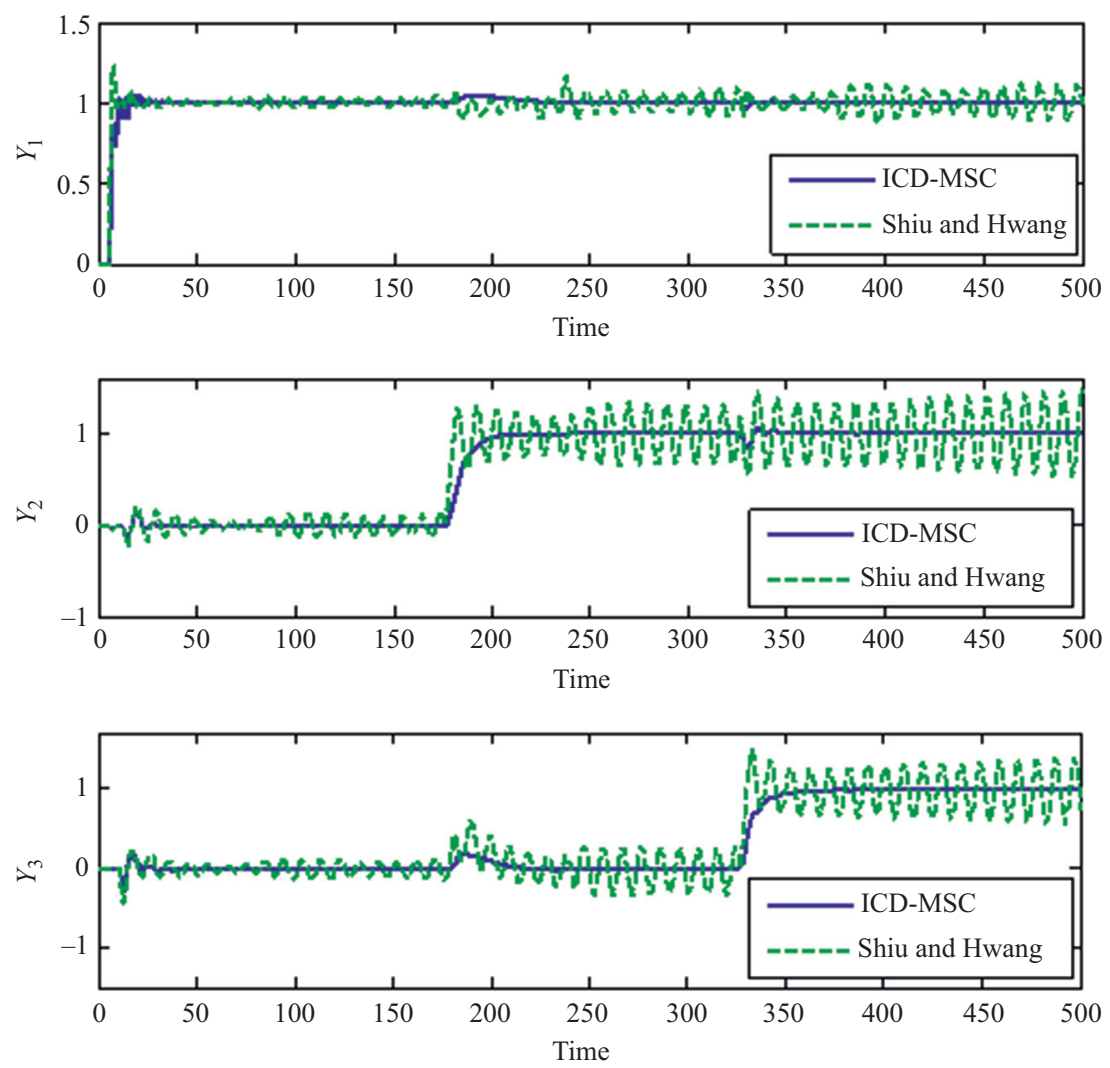

Figure 7 Closed-loop responses at perturbed condition ( $\pm 20 \%$ modeling errors) for Example 2 


\subsection{Process description}

Figure 8 depicts the schematic diagram of a TSCEF system used for bioethanol production. The fermentation of sugarcane molasses by yeast Saccharomyces cerevisiae (or Baker's yeast) takes place within the two bioreactors in series. The bioreactor 2 effluent is sent to a centrifuge unit where the yeast cells (heavy phase) is separated from the liquid containing ethanol (light phase). A small portion of the heavy phase is purged in order to remove the dead cells and other impurities, i.e. to prevent accumulation of impurities. The yeast cells are treated first with dilute sulfuric acid to remove contaminations; water is added to the treatment tank according to a desired ratio $(R)$ of the water flow rate to the total feed flow rate to the bioreactor 1 . Then, the washed yeast cells are recycled to the bioreactor 1. Meanwhile, the light phase from the centrifuge unit is sent to flash vessel unit to partially extract the ethanol in the liquid. A portion of the flash liquid is recycled to the bioreactor 1 , and the rest is taken as product, i.e. sent to distillation columns for further purification. The flash liquid recycle plays an important role which serves to cool down the reactors, thus avoiding the need for heat exchanger installation. The flash liquid recycle is adjusted according to a desired ratio $(r)$ of the flash recycle to the total flash liquid flow rate from the flash vessel unit.

Note that the TSCEF system is difficult to control not only due to the severe process interactions but also because of its nonlinear dynamic behaviors [27]. A number of researchers have studied the control and optimization strategies for the alcoholic extractive fermentation process, for example, Costa et al. [28] studied the control structures for the single-stage alcoholic extractive fermentation process; Sharma and Rangaiah [29] compared the performance of the three-stage fermentation integrated with pervaporation with that of the three-stage fermentation combined with extraction; they found the former to be better; Nandong et al. [30] proposed a PCA-based method for the selection of control structure for the two-stage extractive fermentation process.

The TSCEF system has several numbers of inputs and outputs. However, for the current example, we only consider two inputs and two outputs. The inputs chosen are the flash recycle ratio $(r)$ and the cell recycle ratio $(R)$. The outputs are chosen to be the concentrations of biomass $\left(X_{v}\right)$ and ethanol $\left(E_{t}\right)$ in the second bioreactor. For ethanol production, it is crucial to keep the effluent ethanol concentration variability as small as possible to ensure minimum disturbance to the downstream processing units, i.e. distillation columns.

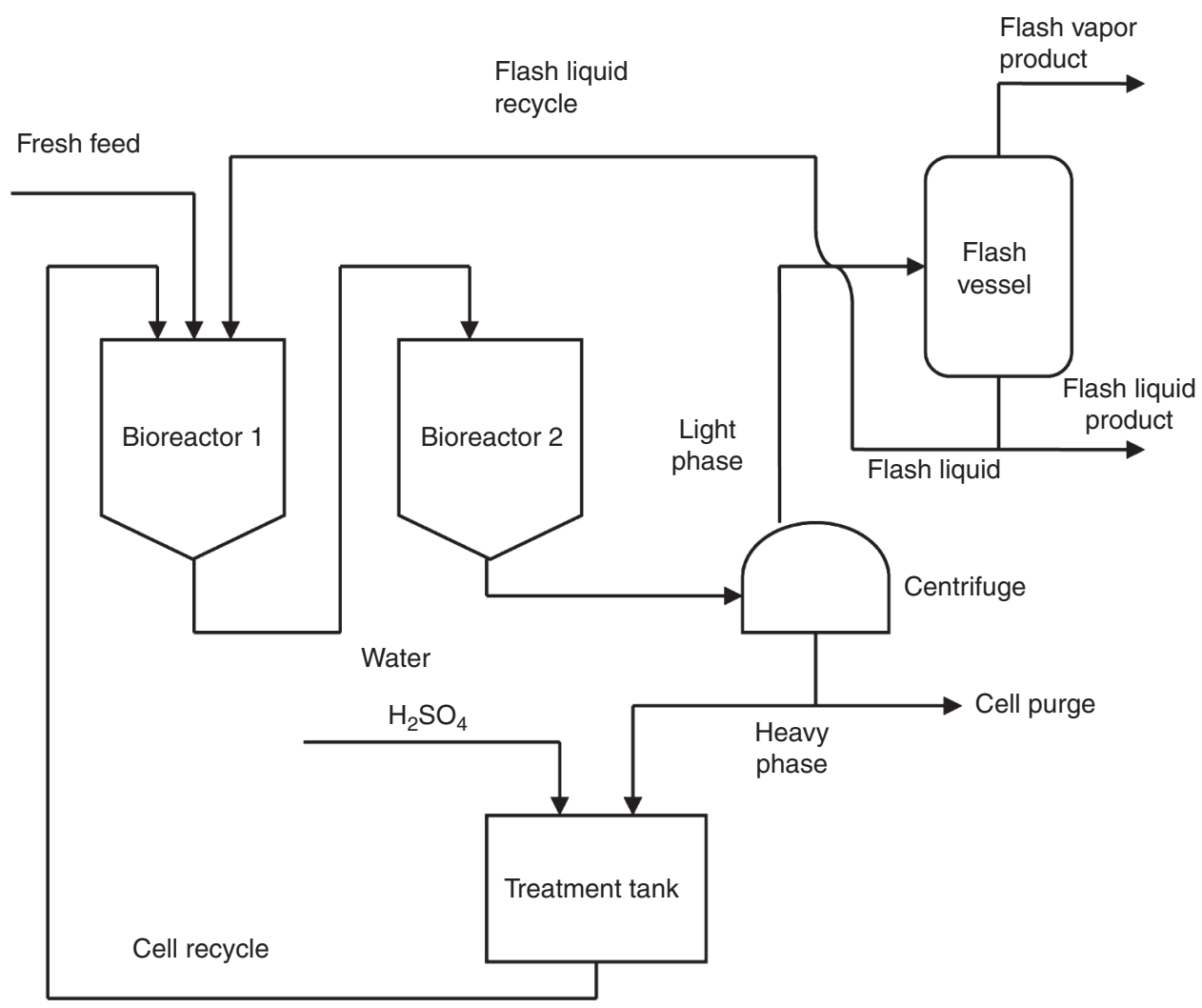

Figure 8 TSCEF system for bioethanol production 


\subsection{Controller design}

A linearized (nominal) model for the TSCEF process is first obtained and given as follows:

$\left[\begin{array}{c}X_{v} \\ E_{t}\end{array}\right]=\left[\begin{array}{cc}\frac{6.77 s^{2}+11.2 s-6.82}{s^{3}+1.49 s^{2}+1.23 s+0.136} & \frac{1.46 s^{2}+42 s-18.8}{s^{3}+3.24 s^{2}+3.13 s+0.423} \\ \frac{-2.25 s^{2}+181.7 s+7.07}{s^{3}+3.08 s^{2}+2.24 s+0.307} & \frac{-3.18 s^{2}+98.4 s-11.4}{s^{3}+2.72 s^{2}+2.1 s+0.304}\end{array}\right]\left[\begin{array}{l}r \\ R\end{array}\right]$

The controller pairings are decided based on the steadystate RGA: the pairings are $r-X_{v}$ for loop 1, and $R-E_{t}$ for loop 2. We compare the performances of three different control schemes: (a) multi-loop PID controllers without decoupling, (2) multi-loop PID controllers with partial decoupling, and (3) 1-way ICD-MSC (I-I structure).

\subsection{Multi-loop PID controllers without decoupling}

The PID controllers are designed using the IMC tuning available in the Matlab SISO Design Tool. The following PID controllers augmented with filters are obtained.

Loop 1

$$
G_{c 1}=\frac{-0.0023\left(6.06 s^{2}+8.13 s+1\right)}{s(0.2 s+1)}
$$

Loop 2

$$
G_{c 2}=\frac{-0.0013\left(6.24 s^{2}+6.4 s+1\right)}{s(0.034 s+1)}
$$

\subsection{Multi-loop PID controllers with partial decoupling}

The decoupling is added from loop 2 to loop 1. As the transfer functions involved possess RHP zeros, we cannot obtain the decoupler in the usual sense as in the case of ideal decoupling technique. Thus, we first factorize the transfer function involved into minimum-phase and nonminimum-phase components. Then, the decoupler is synthesized based on the minimum-phase components. The finalized controller and decoupler tunings are given as follows:

$$
G_{c 1}=\frac{-0.0029\left(6.06 s^{2}+8.13 s+1\right)}{s(0.2 s+1)}
$$

$$
\begin{gathered}
G_{c 2}=\frac{-0.0043\left(6.24 s^{2}+6.4 s+1\right)}{s(0.034 s+1)} \\
D_{12}=\frac{-0.363\left(s^{2}+18.39 s+21\right)}{s^{2}+4.93 s+8.33}
\end{gathered}
$$

\subsection{ICD-MSC scheme (1-way I-I structure)}

The MS predictors are chosen as

$$
\mathbf{W}=\left[\begin{array}{c}
\frac{15.1 s+20.5}{0.95 s^{2}+1.3 s+1} \\
\frac{13.4 s+96.7}{0.61 s^{2}+1.6 s+1}
\end{array}\right]
$$

where the finalized controllers are given by

$$
\mathbf{K}=\left[\begin{array}{cc}
\frac{-0.406(6.7 s+1)}{s} & \frac{-4.12(4.1 s+1)}{s} \\
0.022 & 0.013
\end{array}\right]
$$

The inter-communicative controller is set as $I_{12}=-2$, where the steady-state value is $I_{12}^{S S}=-1.6$. Note that, we choose to set the communication channel from controlloop 2 to control-loop 1 because the inner feedback loop for the loop1 is faster than that of loop 2. If the communication direction is reversed, the inter-communicative scheme becomes less effective.

The performances of the three different control schemes are compared for disturbance rejection. The disturbance considered is the fresh substrate concentration $S_{o}$ : assuming consecutive step changes of magnitudes $-5 \mathrm{~g} / \mathrm{L}$ at $t=5 \mathrm{~h}$ followed by $10 \mathrm{~g} / \mathrm{L}$ at $t=75 \mathrm{~h}$ and finally $-5 \mathrm{~g} / \mathrm{L}$ at $t=150 \mathrm{~h}$. Note that the three control schemes are tested in the nonlinear environment of the two-stage extractive alcoholic fermentation process, i.e. nonlinear dynamic simulation is performed.

Figure 9 shows the comparative regulatory (disturbance rejection) performances of the three different control schemes. Obviously, the proposed ICD-MSC scheme demonstrates superior performance over the multi-loop PID controllers with and without partial decoupling. With respect to multi-loop PID controller with partial decoupling (decoupling PID), the ICD-MSC scheme provides on average about $100 \%$ improved overall performance (in terms of IAE value) over the multi-loop PID with partial decoupling. This example further demonstrates the effectiveness of the proposed ICD-MSC scheme as compared to the conventional control schemes. Interestingly, the ICD-MSC scheme is no more complicated than the multi-loop PID control with decoupling scheme. 

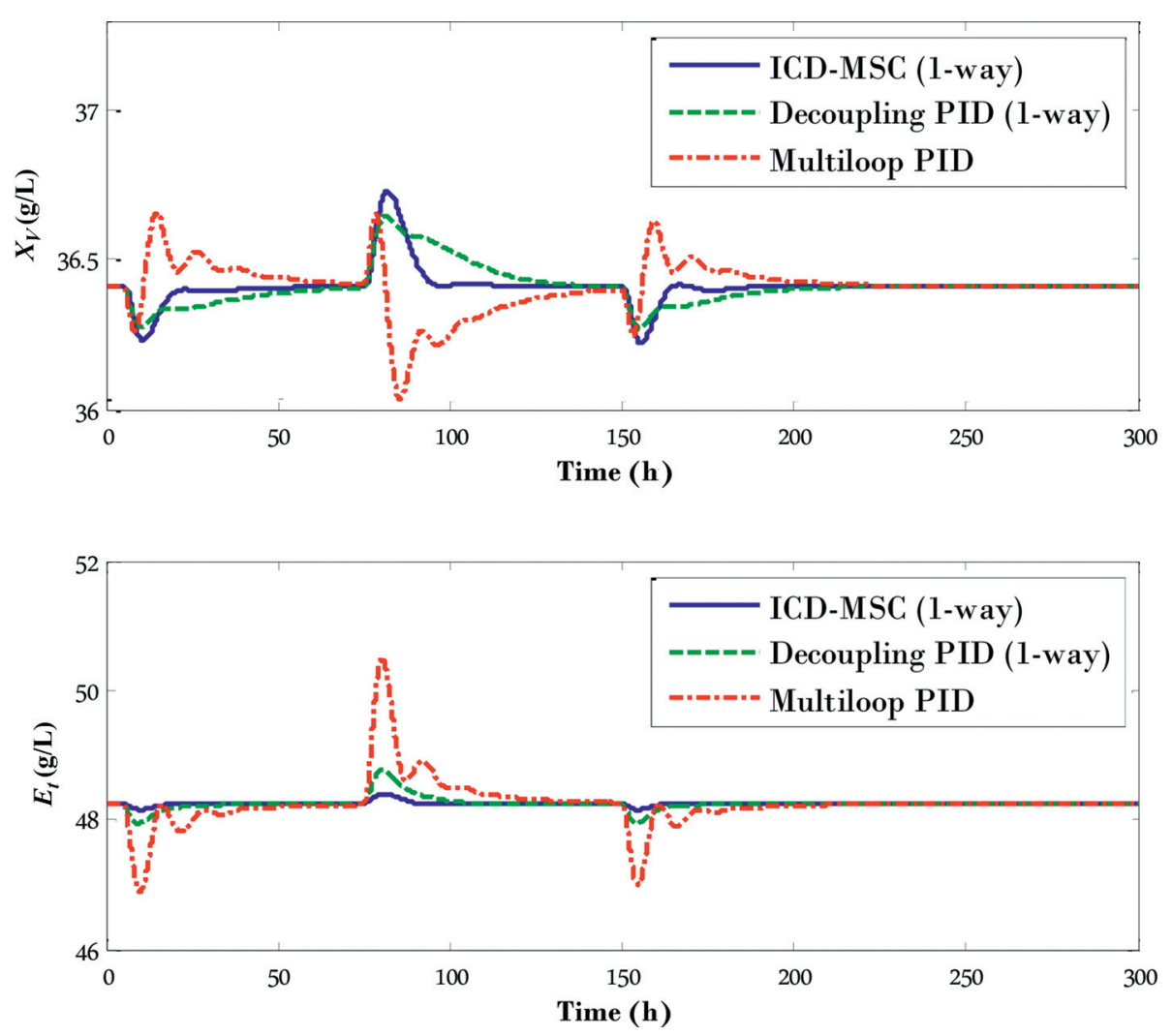

Figure 9 Regulatory responses to consecutive step changes in fresh feed concentration

\subsection{Potential applications of the ICD-MSC scheme}

The proposed ICD-MSC scheme represents a new approach to overcoming poor control performance due to process interactions. Since the scheme uses the concept of fast feedback control action as a way to mitigate the adverse effect of loop interactions, this scheme is more likely to be less vulnerable to the modeling errors than the conventional decoupling schemes. Note that the performance of conventional decoupling schemes is often seriously degraded by modeling errors because the schemes use feedforward control action as a way to mitigate the effect of loop interactions.

It is worth highlighting that the proposed ICD-MSC scheme can be used to control critical processes in complex chemical or bio-chemical plants, such as the heat integrated distillation columns, series reactors or bioreactors with mass-energy recycles, and multiple-effect evaporators. Additionally, the proposed ICD-MSC scheme can also be used to design multi-loop PID control system augmented with decoupling controllers; the application of superposition principle to the ICD-MSC structure yields separate multi-loop PID control and decoupling systems (this extension will be reported in future work). In this case, the ICD-MSC can be used as an alternative way to design physically realizable decoupling controllers. Note that, in many cases the conventional decoupling methods may fail to yield physically realizable decoupling controllers, which often results in ad hoc approximations of the decouplers used.

\section{Conclusions}

A new decentralized control scheme, namely the ICDMSC based on multiple single MS controllers has been presented. This new scheme is able to improve the performance of MIMO control in the presence of strong process interactions. The MS controllers have several advantages over the conventional PID controllers: (1) it is able to provide improved nominal performance and performance robustness in the presence of long timedelays/slow RHP zeros, and (2) the inner-layer feedback loop of a given MS controller can be used to reject the disturbance from another controller due to loop interaction. As the proposed scheme rejects the disturbance (due to loop interaction) via a fast feedback control 
rather than via feedforward control as in the conventional decoupling schemes, the proposed scheme tends to be more robust against modeling errors than the conventional decoupling control schemes, which has confirmed by extensive simulation study. Future work will include the development of a more rigorous design of the inter-communicative controllers based on solving problems $P_{1}$ to $P_{4}$ for both ICD-MSC structures. The work on developing a hybrid decoupling and intercommunicative scheme will also be investigated in future. In view that many industrial processes are non-square, we will also address the extension of the current ICD-MSC scheme to such non-square systems as part of the future study.

Acknowledgment: This work is partially supported by Curtin Sarawak Research Cluster Fund under the auspices of Intelligent Systems, Design and Control (ISDC) Research Area at Curtin University, Malaysia.

\section{References}

1. Lee J, Cho W, Edgar TF. Multiloop PI controller tuning for interacting multivariable processes. Comput Chem Eng 1998;22:1711-23.

2. Huang HP, Jeng JC, Chiang $\mathrm{CH}$, Pan W. A direct method for multi-loop PI/PID controller design. J Process Control 2003;13:769-86.

3. Luyben WL. Simple method for tuning SISO controllers in multivariable systems. Ind Eng Chem Process Des Dev 1986;25:654-60.

4. Ziegler JG, Nichols NB. Optimum settings for automatic controllers. Trans ASME 1942;64:759-68.

5. Lee J, Edgar TF. Multiloop PI/PID control system improvement via adjusting the dominant pole or the peak amplitude ratio. Chem Eng Sci 2006;61:1658-66.

6. Mayne DQ. The design of linear multivariable systems. Automatica 1973;9:201-7.

7. Hovd M, Skogestad S. Sequential design of decentralized controllers. Automatica 1994;30:1601-7.

8. Shiu SJ, Hwang SH. Sequential design method for multivariable decoupling and multiloop PID controllers. Ind Eng Chem Res 1998;37:107-19.

9. Loh A, Hung C, Quek C, Vasnani V. Auto-tuning of multi-loop proportional-integral controllers using relay feedback. Ind Eng Chem Res 1993;32:1102-7.

10. Grosdidier P, Morari M. Interaction measures for systems under decentralized control. Automatica 1986;22:309-20.

11. Vu T, Lee M. Independent design of multi-loop PI/PID controllers for interacting multivariable processes. J Process Control 2010;20:922-33.

12. Jung J, Choi J, Lee J. One-parameter method for a multiloop control system design. Ind Eng Chem Res 1999;38:1580-8.

13. Chen D, Seborg D. Design of decentralized PI control systems based on Nyquist stability analysis. J Process Control 2003;13:27-39.

14. Lee M, Lee K, Kim C, Lee J. Analytical design of multi-loop PID controllers for desired closed-loop responses. AIChE J 2004;50:1631-5.

15. Astrom K, Hagglund T. PID controllers: theory, design, and tuning, 2nd ed. Research Triangle Park, NC: Instrument Society of America, 1995.

16. Yu C. Autotuning of PID controllers: relay feedback approach. New York: Springer, 1999.
17. Shinskey FG. Process control systems: application, design and adjustment, 4th ed. New York: McGraw-Hill, 1996.

18. Gagnon E, Pomerleau A, Desbiens A. Simplified, ideal or inverted decoupling. ISA Trans 1998;37:265-76.

19. Waller K. Decoupling in distillation. AIChE J 1974;20:592-4.

20. Garrido J, Vazquez F, Morilla F. Centralized multivariable control by simplified decoupling. J Process Control 2012;22:1044-62.

21. Rajapandiyan C, Chidambaram M. Controller design for MIMO processes based on simple decoupled equivalent transfer functions and simplified decouplers. Ind Eng Chem Res 2012;51:12398-410.

22. Garrido J, Vazquez F, Morilla F. An extended approach of inverted decoupling. J Process Control 2011;21:55-68.

23. Chen P, Zhang W. Improvement on an inverted decoupling technique for a class of stable linear multivariable processes. ISA Trans 2007;46:199-210.

24. Nandong J, Zang Z. Novel multiscale control scheme for nonminimum-phase processes. Ind Eng Chem Res 2013;52:8248-59.

25. Nandong J, Zang Z. High-performance multi-scale control scheme for stable, integrating and unstable time-delay processes. J Process Control 2013;23:1333-43.

26. Chien IL, Huang HP, Yang JC. A simple multi-loop tuning method for PID controllers with no proportional kick. Ind Eng Chem Res 1999;38:1456-68.

27. Nandong J, Samyudia Y, Tade MO. Dynamic simulation and optimization of two-stage extractive alcoholic fermentation process: design impact on controllability. Chem Prod Process Model 2006;1:1934-2659.

28. Costa AC, Atala DI, Maugeri F, Maciel R. Factorial design and simulation for the optimization and determination of control structures for an extractive alcoholic fermentation. Process Biochem 2001;37:125-37.

29. Sharma S, Rangaiah GP. Modeling and optimization of a fermentation process integrated with cell recycling and pervaporation for multiple objectives. Ind Eng Chem Res 2012;51:5542-51.

30. Nandong J, Samyudia Y, Tade MO. Novel PCA-based technique for identification of dominant variables for partial control. Chem Prod Process Model 2010;5: 1934-2659. 